Published in final edited form as:

Lancet. 2014 February 15; 383(9917): 614-621. doi:10.1016/S0140-6736(13)62302-8.

\title{
Medical management with or without interventional therapy for unruptured brain arteriovenous malformations (ARUBA): a multicentre, non-blinded, randomised trial
}

\begin{abstract}
Prof J P Mohr, MD*, Prof Michael K Parides, PhD*, Prof Christian Stapf, MD*, Ellen Moquete, RN, Claudia S Moy, PhD, Jessica R Overbey, MS, Prof Rustam Al-Shahi Salman, FRCP, Prof Eric Vicaut, MD, Prof William L Young, $M^{\dagger}{ }^{\dagger}$, Prof Emmanuel Houdart, MD, Prof Charlotte Cordonnier, MD, Prof Marco A Stefani, MD, Andreas Hartmann, MD, Prof Rüdiger von Kummer, MD, Prof Alessandra Biondi, MD, Prof Joachim Berkefeld, MD, Catharina J M Klijn, MD, Kirsty Harkness, MD, Richard Libman, MD, Xavier Barreau, MD, and Prof Alan J Moskowitz, MD for the international ARUBA investigators $\$$

The Neurological Institute, Columbia University Medical Center, New York, NY, USA (Prof J P Mohr MD, Prof C Stapf MD); International Center for Health Outcomes and Innovation Research, Department of Health Evidence and Policy, Icahn School of Medicine at Mount Sinai, New York, NY, USA (Prof M K Parides PhD, E Moquete RN, J R Overbey MS, Prof A J Moskowitz MD); Department of Neurology (Prof C Stapf), DHU NeuroVasc (Prof C Stapf), Department of Neuroradiology (Prof E Houdart MD), Unité de Recherche Clinique (Prof E Vicaut MD), APHPHôpital Lariboisière, Univ Paris Diderot-Sorbonne Paris Cité, Paris, France; National Institute of Neurological Disorders and Stroke, National Institutes of Health, Bethesda, MD, USA (C S Moy $\mathrm{PhD}$ ); Division of Clinical Neurosciences, Centre for Clinical Brain Sciences, University of Edinburgh, Edinburgh, UK (Prof R AI-Shahi Salman FRCP); Department of Anesthesia and Perioperative Care, University of California, San Francisco, CA, USA (Prof W L Young MD); Department of Neurology, CHRU Lille, Université Lille Nord de France, Lille, France (Prof C Cordonnier MD); Department of Neurology and Neurosurgery, Hospital de Clinicas de Porto Alegre, Porto Alegre, Brazil (Prof M A Stefani MD); Charité-Universitätsmedizin Berlin Berlin, Germany (A Hartmann MD); Department of Neurology, Klinikum Frankfurt/Oder, Frankfurt/Oder
\end{abstract}

Correspondence to: Prof Christian Stapf, Neurovascular Unit, Department of Neurology, APHP-Hôpital, Lariboisière, 75175 Paris cedex 10, France, christian.stapf@1rb.aphp.fr.

Contributed equally

†Prof Young died in August, 2013

¥Members listed in appendix

See Online for appendix

Contributors

JPM contributed to study design, scientific literature search, data collection, data analysis, data interpretation, figures, tables, writing, and editing. MKP contributed to study design, final data analysis, data interpretation, figures, tables, writing, and editing. CS contributed to study design, scientific literature search, data collection, data analysis, data interpretation, figures, tables, first manuscript draft, writing, and editing. EM contributed to data monitoring, data interpretation, figures, tables, writing, and editing. CSM contributed to study design, scientific literature search, data interpretation, figures, tables, writing, and editing. JRO contributed to data analysis, data interpretation, figures, and tables. RA-SS contributed to scientific literature search, data collection, data interpretation, tables, writing, and editing. EV contributed to study design, scientific literature search, data monitoring, data interpretation, figures, tables, writing, and editing. WLY contributed to study design, literature search, data collection, data interpretation. $\mathrm{EH}, \mathrm{CC}, \mathrm{MAS}, \mathrm{AH}, \mathrm{RvK}, \mathrm{AB}, \mathrm{JB}, \mathrm{CJMK}, \mathrm{KH}, \mathrm{RL}$, and $\mathrm{XB}$ contributed to data collection, data interpretation, and editing. AJM contributed to study design, data analysis, data interpretation, figures, tables, writing, and editing.

Conflicts of interest

We declare that we have no conflicts of interest. 
Oder, Germany (A Hartmann); Department of Neuroradiology, University Hospital Dresden, Dresden, Germany (Prof R von Kummer MD); Department of Neuroradiology and Endovascular Therapy, Jean Minjoz Hospital, University of Franche Comté, Besançon, France (Prof A Biondi MD); Department of Neuroradiology, Universitätsklinikum Frankfurt, Frankfurt am Main, Germany (Prof J Berkefeld MD); Department of Neurology and Neurosurgery, Brain Center Rudolf Magnus, University Medical Center, Utrecht, Netherlands (C J M Klijn MD); Department of Neurology, Royal Hallamshire Hospital, Sheffield, UK (K Harkness MD); Department of Neurology, North Shore Long Island Jewish Medical Center, New Hyde Park, NY, USA (R Libman MD); and Department of Diagnostic and Interventional Neuroimaging, CHU Pellegrin, Bordeaux, France (X Barreau MD)

\section{Summary}

Background-The clinical benefit of preventive eradication of unruptured brain arteriovenous malformations remains uncertain. A Randomised trial of Unruptured Brain Arteriovenous malformations (ARUBA) aims to compare the risk of death and symptomatic stroke in patients with an unruptured brain arteriovenous malformation who are allocated to either medical management alone or medical management with interventional therapy.

Methods-Adult patients ( $\geq 18$ years) with an unruptured brain arteriovenous malformation were enrolled into this trial at 39 clinical sites in nine countries. Patients were randomised (by webbased system, in a 1:1 ratio, with random permuted block design [block size 2, 4, or 6], stratified by clinical site) to medical management with interventional therapy (ie, neurosurgery, embolisation, or stereotactic radiotherapy, alone or in combination) or medical management alone (ie, pharmacological therapy for neurological symptoms as needed). Patients, clinicians, and investigators are aware of treatment assignment. The primary outcome is time to the composite endpoint of death or symptomatic stroke; the primary analysis is by intention to treat. This trial is registered with ClinicalTrials.gov, number NCT00389181.

Findings-Randomisation was started on April 4, 2007, and was stopped on April 15, 2013, when a data and safety monitoring board appointed by the National Institute of Neurological Disorders and Stroke of the National Institutes of Health recommended halting randomisation because of superiority of the medical management group ( $\log$-rank $Z$ statistic of $4 \cdot 10$, exceeding the prespecified stopping boundary value of 2.87). At this point, outcome data were available for 223 patients (mean follow-up 33.3 months [SD 19.7]), 114 assigned to interventional therapy and 109 to medical management. The primary endpoint had been reached by $11(10 \cdot 1 \%)$ patients in the medical management group compared with $35(30.7 \%)$ in the interventional therapy group. The risk of death or stroke was significantly lower in the medical management group than in the interventional therapy group (hazard ratio $0 \cdot 27,95 \%$ CI $0 \cdot 14-0 \cdot 54$ ). No harms were identified, other than a higher number of strokes ( $45 v s 12, \mathrm{p}<0.0001)$ and neurological deficits unrelated to stroke (14 vs $1, \mathrm{p}=0 \cdot 0008)$ in patients allocated to interventional therapy compared with medical management.

Interpretation-The ARUBA trial showed that medical management alone is superior to medical management with interventional therapy for the prevention of death or stroke in patients with unruptured brain arteriovenous malformations followed up for 33 months. The trial is 
continuing its observational phase to establish whether the disparities will persist over an additional 5 years of follow-up.

Funding - National Institutes of Health, National Institute of Neurological Disorders and Stroke.

\section{Introduction}

Brain arter iovenous malformations are diagnosed most often in adults aged about 40 years. Haemorrhage was the usual means of discovery before non-invasive imaging, but in the past three decades such imaging has helped with the detection of brain arteriovenous malformations and the proportion being diagnosed unruptured has almost doubled. ${ }^{1,2}$

An earlier retrospective series ${ }^{3}$ estimated a $4 \%$ crude annual rupture rate for brain arteriovenous malformations, but this risk was derived from combined outcomes, including those already having bled. More recent prospective studies ${ }^{4,5}$ report bleeding rates as low as $1 \%$ per year for those discovered unruptured. Furthermore, first haemorrhage syndromes are often mild, with bleeding often mainly confined to the brain arteriovenous malformation itself or originating from the venous side of the malformation. ${ }^{6,7}$ Approaches to eradicate a brain arteriovenous malformation, bled or not, include various treatment techniques (neurosurgery, endovascular embolisation, and stereotactic radiotherapy) used alone or in combination with varying degrees of treatment-associated morbidity and mortality. 8,9

In the past decade, debates have addressed whether preventive lesion eradication offers a clinical benefit for patients diagnosed with an unruptured brain arteriovenous malformation. ${ }^{10,11}$ A Randomised trial of Unruptured Brain AVMs (ARUBA) was organised to address this clinically compelling question.

\section{Methods}

\section{Study design and participants}

ARUBA is a prospective, multicentre, parallel design, non-blinded, randomised controlled trial involving 39 active clinical sites in nine countries (appendix). Site selection was based on centre experience with management of at least ten brain arteriovenous malformations per year, presence of a multidisciplinary arteriovenous malformations treatment team, and documented academic interest in clinical brain arteriovenous malformation research.

We compare the risk of death and symptomatic stroke in patients with an unruptured brain arteriovenous malformation who are allocated to either medical management alone or medical management with planned efforts at eradication of the brain arteriovenous malformation with interventional therapy. The three specific aims of the trial are: 1) to establish whether medical management is superior to interventional therapy for prevention of the composite outcome of death from any cause or symptomatic stroke in the management of unruptured brain arterio venous malformations; 2) if medical management is not superior to interventional therapy, to establish whether medical management is not inferior to interventional therapy for prevention of the composite outcome of death from any cause or symptomatic stroke in the management of unruptured brain arteriovenous malformations; 3) to establish whether treatment of unruptured brain arteriovenous 
malformations by medical management decreases the risk of death or clinical impairment (modified Rankin scale score of 2 or higher) at 5 years' post-randomisation compared with interventional therapy.

All patients aged 18 years or older with an unruptured brain arteriovenous malformation diagnosed by appropriate neurovascular imaging (eg, by catheter angiography, MRI or MR angiography, CT or CT angiography, at the discretion of local ARUBA investigators) were deemed potential candidates for this trial. The main exclusion criteria were any imaging evidence of previous haemorrhage from a brain arteriovenous malformation, any previous interventional treatment attempt, or presence of a brain arteriovenous malformation deemed unsuitable for attempted eradication by the local centre (full exclusion criteria are listed in the appendix). All variables relating to brain arteriovenous malformations (including the definition of clinical and morphological baseline characteristics) used in the trial are defined according to currently recommended reporting terminology for clinical brain arteriovenous malformation research. ${ }^{2,12}$

The trial is monitored by an independent data and safety monitoring board (appendix) appointed by the National Institute of Neurological Disorders and Stroke (NINDS) of the National Institutes of Health (NIH); the protocol and consent forms were approved by the relevant institutional review boards or equivalent ethics committees at the 61 international institutions in contract, and all participants gave written informed consent before randomisation.

\section{Randomisation and masking}

Randomisation was done centrally through a web-based system that confirmed eligibility before issuing a treatment assignment. Patients were assigned in a 1 to 1 ratio (random permuted block design using blocks of size 2,4 , or 6 , randomly selected with equal probability, stratified by clinical site) to medical management alone or medical management with interventional therapy. Assignments are not masked to participants, clinicians, or investigators. A senior study neurologist who is not involved in provision of the interventional procedures performs the clinical outcome assessment. All primary and secondary outcome events and imaging studies are assessed by an independent multidisciplinary committee of international adjudicators (appendix).

\section{Procedures}

For patients allocated to the interventional therapy group, interventional therapy includes any neurosurgical, endovascular, or radiotherapy procedure (single or multiple) deemed appropriate by local ARUBA investigators to achieve complete eradication of the brain arteriovenous malformation. Medical management is irrespective of randomisation assignment, because all participants receive pharmacological therapy for existing medical disorders (eg, seizures, headaches) or any coexisting vascular risk factors (diabetes, arterial hyper tension) as needed. Patients in the medical management group who reach the primary endpoint (ie, who develop a symptomatic stroke) related to their brain arteriovenous malformation become candidates for the same interventional options as those randomised to the interventional therapy group but do not count as crossovers. Patients allocated to medical 
management who subsequently receive interventional therapy are deemed to have crossed over if the reason for intervention was other than stroke related to their brain arteriovenous malformation. All patients who switch from interventional therapy to medical management after randomisation are defined as crossovers.

In-person neurological follow-up visits are scheduled at 6 month intervals during the first 2 years and annually, with telephone contacts every 6 months thereafter. At each visit or telephone contact, patients are actively screened for the possibility of new stroke, neurological deficits, seizures, headaches, or any other clinically important event. Clinical standard scales (modified Rankin scale, NIH stroke scale) and health-related quality-of-life measures (EuroQoL, SF-36) ${ }^{13}$ are systematically documented at every follow-up visit.

Baseline imaging (MRI or MR angiography, CT or CT angiography, or catheter angiography) is collected after enrolment for each patient, confirmed by a credentialed senior neuroradiologist on site. Additionally, all baseline imaging studies are subject to independent centralised adjudication for diagnostic accuracy. Lesion eradication is confirmed on the basis of catheter angiography and central adjudication. Any imaging studies related to neurological adverse events are systematically collected in electronic format and included in the material for independent clinical event adjudication.

The primary outcome is time to the composite event of death from any cause or symptomatic stroke. Stroke is defined as a clinically symptomatic event (any new focal neurological deficit, seizure, or new-onset headache) that is associated with imaging findings of haemorrhage or infarction. Haemorrhage is defined as fresh intracranial blood on head CT or MRI, or in the cerebrospinal fluid. Infarction is defined as a new ischaemic lesion on cranial CT or MRI (diffusion-weighted, T2-weighted, or fluid-attenuated inversion recovery MRI). The secondary outcome is clinical impairment at 5 years with a modified Rankin scale score of 2 or higher. ${ }^{14}$

\section{Statistical analysis}

The design for ARUBA originally approved by the NIH/NINDS Study Section was for 800 patients to be randomised in equal allocation to interventional therapy procedures (standard treatment) versus medical management alone (experimental study group). This design, based on an assumed 5-year event rate of $12 \%$ for patients treated with medical management alone and $22 \%$ with interventional therapy, offered a statistical power of $87.5 \%$ to detect a $40 \%$ reduction in the hazard for death or symptomatic stroke over 5 years. Because of lower than expected accrual rates after 18 months of randomisation, the data and safety monitoring board accepted a revised design from the study statistician investigators. The sample size of 400 reduces the trial's statistical power to $80 \%$ to detect a $46 \%$ reduction in the hazard for death or symptomatic stroke for patients in the medical management group.

Two interim analyses were scheduled and undertaken, based on a group sequentialmonitoring procedure with stopping rules established using a Lan-DeMets stopping boundary and an O'Brien-Fleming spending function. The primary null hypothesis was that no difference existed in the risk of symptomatic stroke or death between patients randomised to medical management compared with patients randomised to interventional therapy. The 
primary null hypothesis was tested in an intention-to-treat (as-randomised) analysis using a 0.05 level log-rank test. We also present results of an as-treated analysis, with patients who crossed over analysed according to the type of management they eventually received.

Cumulative stroke-free survival curves for each group were estimated by the Kaplan-Meier method, and Cox proportional-hazards regression models were used to estimate hazard ratios (HRs; instantaneous risk). We tested the proportionality of hazards for the interaction between treatment and the log of time and did not identify any departure from the proportionality assumption. Cox regression was also used to assess the interaction of treatment with five prespecified covariates; Spetzler-Martin grade, ${ }^{15}$ location of brain arteriovenous malformation, venous drainage pattern, age, and time from discovery of brain arteriovenous malformation. Risk of clinical impairment is based on the proportion of patients with a modified Rankin scale score of 2 or higher, and is compared between groups using a $\chi^{2}$ test. Group differences in the incidence of adverse events including all strokes, focal deficits, seizures, and headaches were compared using Poisson regression.

The trial is registered with ClinicalTrials.gov, number NCT00389181.

\section{Role of the funding source}

The funder of the study participated in study design, data interpretation, and writing of the report, but had no role in data collection or data analysis. All authors had access to the data in the study, and the corresponding author had final responsibility for the decision to submit for publication.

\section{Results}

We randomised 226 patients during the period from April 4, 2007, to April 15, 2013, at a mean rate of 3.2 patients per month (appendix). Figure 1 shows the trial profile. We had screened a further 1514 patients for eligibility, of whom 1014 were ineligible for enrolment, largely because of evidence of previous haemorrhage or a history of previous treatment. Of the 500 patients deemed eligible, 323 refused participation in the trial, and 42 were managed by centres that randomised no patients. For the 361 patients seen at active centres, 226 $(63 \%)$ were randomised, $74(20 \%)$ underwent interventional management outside the trial, and $61(17 \%)$ underwent medical management outside the trial or were reported with undecided status. No information on outcome is available for any patients managed outside the trial.

On April 15, 2013, randomisation was halted by the NINDS/NIH at the recommendation of the data and and monitoring board. This decision was based on the results of the second planned interim analysis of 223 patients with mean follow-up of 33.3 months (SD 19.7; median $33 \cdot 2$ months, IQR $16 \cdot 3-49 \cdot 8$ ), representing $53 \%$ of the anticipated outcome information. The results of the interim analysis showed efficacy of medical management for the prevention of death or stroke, with an observed log-rank $Z$ statistic of 4.10, exceeding the prespecified stopping boundary value of $2 \cdot 87$.

116 patients were randomised to interventional therapy, and 110 to medical management; data for two patients in the interventional therapy group and one patient in the medical 
management were excluded from the analyses because randomisation occurred after the dataset had been locked. Of the 223 patients with data available, $41 \%$ were women and the mean age of enrolees was 44.5 years (SD 12.3, range 20-69; table $1^{12,15}$ ). $83 \%$ of enrollees were white, $7 \%$ were African American, and $12 \%$ identified their ethnicity as Hispanic. The diameter of all randomised brain arteriovenous malformations was less than $60 \mathrm{~mm}(<30$ $\mathrm{mm}, \mathrm{n}=138 ; 30-60 \mathrm{~mm}, \mathrm{n}=85$ ). None of the patients had a Spetzler-Martin Grade score higher than 4; most patients (62\%) had scores of 2 or less. Complying with entry criteria, all patients had a modified Rankin scale score of 1 or lower.

Baseline demographic, clinical, and lesion characteristics and modified Rankin scale scores were much the same between groups except for minor imbalances tending towards higher proportions of small $(<3 \mathrm{~cm})$ brain arteriovenous malformations and of patients with baseline neurological symptoms in the interventional therapy group (table 1). At the time of the analysis, length of follow-up was balanced between interventional therapy (mean 32.9 months, SD 19.2) and medical management (33.7 months, 20.2); overall data completeness was $98 \%$.

For patients allocated to interventional therapy, brain arteriovenous malformations were treated by neurosurgery alone $(n=5)$, embolisation alone $(n=30)$, or radiotherapy alone $(n=31)$, or using a multimodal approach combining embolisation with either neurosurgery $(\mathrm{n}=12)$, radiotherapy $(\mathrm{n}=15)$, or both $(\mathrm{n}=1)$. At the time of the analysis, 53 patients randomised to interventional therapy had ongoing treatment plans, whereas 20 had not yet initiated therapy. The median time to first intervention was 76 days (IQR 45-119; mean 106, SD 122) after randomisation (appendix). Seven patients allocated to medical management received interventional therapy without rupture of previous brain arteriovenous malformation (figure 1) and were deemed crossovers in the as-treated analysis. Three of those randomised to interventional therapy had an outcome event before initiation of interventional therapy and were included in the medical management group for the astreated analysis. Seven patients (five in the interventional therapy group, two in the medical management group) discontinued their participation in the trial (3\%) during follow-up.

Tables 2 and 3 show the primary outcome events. A primary event had occurred for 11 $(10.1 \%)$ of the 109 patients randomised to medical management versus $35(30.7 \%)$ of the 114 patients randomised to interventional therapy (as-randomised analysis). An intention-totreat analysis of the primary endpoint (time to first stroke or death) showed that the hazard (instantaneous risk) was significantly less in the medical management group (HR 0.27, 95\% CI 0.14-0.54) as compared with the interventional therapy group ( $<<0.0001$; figure $2 \mathrm{~A}$ ). The effect was stronger in the astreated analysis (HR $0 \cdot 19,95 \%$ CI $0 \cdot 09-0 \cdot 38, \mathrm{p}<0 \cdot 0001$; figure 2B). Adjustment for the minor imbalances identified in the baseline characteristics had no effect on the significance of the primary endpoint analysis (data not shown).

The disparity of primary event rates was associated with a substantially better functional outcome in the medical management group: the risk of death and neurological disability (modified Rankin scale $\geq 2$ ) at 30 months was significantly lower for medical management (eight of $53,15.1 \%$ ) than for interventional therapy ( 24 of $52,46.2 \%$; relative risk [RR] $0 \cdot 33,95 \%$ CI 0.16-0.66) in an as-randomised analysis. This effect remained stable to 36 
months for medical management (six of 43, 14.0\%) compared with interventional therapy (17 of $44,38 \cdot 6 \%$; RR $0 \cdot 36,95 \%$ CI 0·16-0.83). No unexpected harms were identified, other than a higher proportion of strokes (ie, including recurrent strokes documented after a primary endpoint had been reached) and other neurological deficits (unrelated to stroke) in patients allocated to interventional therapy (asrandomised analysis; table 4). In a predefined secondary analysis, primary outcome event rates in the medical management group were low across all Spetzler-Martin grades, but events for the interventional therapy group were significantly more frequent in grades II and III in both the as-randomised and as-treated analyses (appendix).

\section{Discussion}

In this study of 223 patients with unruptured brain arteriovenous malformations, the risk of death or stroke was significantly lower in the medical management group than in the interventional therapy group after a mean follow-up of about 33 months. Patients followed up without intervention also had a significantly lower risk of death and neurological disability (modified Rankin scale 2 ) than those in the interventional group. No unexpected harms were identified, other than a higher proportion of strokes and neurological deficits unrelated to stroke in patients allocated to interventional therapy compared with medical management. Both interventional eradication and conservative follow-up are recognised management strategies in patients with brain arteriovenous malformations. ${ }^{16,17}$ As far as we are aware, ARUBA is the first randomised controlled clinical trial assessing the outcomes from these two management strategies (ie, medical management with interventional therapy or medical management alone) in patients diagnosed with an unruptured brain arteriovenous malformation (panel). ${ }^{18}$

The trial has shown significant results, and earlier than expected: a more than threefold increased risk of stroke and death after the initiation of interventional therapy compared with medical management alone in patients with an unruptured brain arteriovenous malformation (HR 3.70, 95\% CI 1.85-7.14, as randomised). The findings are in line with population-based data ${ }^{19}$ that suggested a significantly increased risk of stroke and focal neurological deficits for unruptured brain arteriovenous malformation in patients undergoing an intervention (HR $2 \cdot 53,95 \%$ CI 1.06-6.04). In our study, the incidence of primary outcome events did not differ between participating sites in different countries (appendix). Also, the proportion of outcome events we identified for interventional therapy (30.7\%, as randomised) closely approximate interventional complication rates cited in a systematic meta-analysis ${ }^{8}$ of brain arteriovenous malformation treatment for both bled and unbled lesions: surgery (29\%, range $1 \cdot 5-54$ ), embolisation (25\%, range 7.6-55), and radiotherapy (13\%, range 0-63).

For patients followed up without interventional therapy, ARUBA confirms a low spontaneous rupture rate of $2.2 \%$ per year (95\% CI $0.9-4.5)$. These natural history data corroborate earlier reports,,${ }^{4,5}$ even those from cohorts including cases having bled, ${ }^{20}$ and our secondary analyses argue against a predictive spontaneous haemorrhage effect from the Spetzler-Martin Grade. ${ }^{9}$ 
No randomised trial has ever been truly populationbased, and few have comparable representation from individual centres, ${ }^{21}$ making debates over credibility of outcome data common. ${ }^{22,23}$ The proportion of randomised cases $(n=226$ overall) versus screened patients $(\mathrm{n}=1740)$ in our study might seem low $(13 \%)$, but is actually higher than in previous interventional stroke prevention trials ${ }^{24}$ assessing elective extracranial vascular surgery 25 (randomised $4 \%$ ) and intracranial vascular surgery ${ }^{26}(4 \%)$. Additionally, actively randomising ARUBA sites enrolled an unusually large proportion (63\%) of eligible patients. Voluntary participation explains the refusal of randomisation by some potentially eligible patients, and many factors contribute to management decisions that keep eligible patients outside any trial. Potentially useful collection of outcome data for patients deemed eligible but not enrolled in ARUBA was not realised: no centres responded to offers circulated by the study organisers to create a registry for such outcome data. For actual trial participants, the baseline profile is internally balanced and supports the fairness of the comparison made (table 1). Inclusion of a broad international panel of multidisciplinary treatment teams assured representativeness of the cohort and presence of the whole range of welldocumented interventional treatment strategies for brain arteriovenous malformations. When compared with the most recent population-based data for brain arteriovenous malformations from Scotland ${ }^{19}$ and Finland ${ }^{20}$ the profiles of patients in ARUBA show smaller lesion size and lower Spetzler-Martin scale scores (appendix), suggesting that patients recruited to ARUBA represent an optimum population for interventional therapy. The favourable comparison with representative patient cohorts, along with similar primary outcome event rates seen across different countries (appendix) support the external validity of the trial and the generalisability of its results.

As a randomised clinical trial, ARUBA features adjudicated primary outcome assessment, high data quality (98\% data completeness), and strong patient adherence (only 3\% lost to follow-up despite the trial's long-term design). Also, the objective documentation of primary outcome events (ie, death or symptomatic stroke confirmed by imaging) is unlikely to be biased by its non-blinded design. The reliability of the primary outcome analysis is further emphasised by the steady accumulation of secondary (neurological disability) outcome variables. The mandated early end to randomisation limits the power of predefined secondary analyses regarding potential predictors of both brain arteriovenous malformation natural history and treatment risk. With regard to interventional treatment, no standard management plans have been promulgated by speciality groups so $\mathrm{far}^{16}$ and investigators insisted on the freedom to utilise their expertise in the same fashion as pursued in their usual practice, much the same as in previous large interventional trials. ${ }^{27,28}$ Since many patients in the interventional group are still undergoing therapy for brain arteriovenous malformation, a comprehensive analysis of the technical and clinical treatment success by treatment modality is still premature at this stage. Furthermore, neither the original nor the final sample size are powered for assessment of outcome by interventional treatment types.

The planned long-term follow-up for ARUBA will establish whether the disparity in outcome events identified up to now will persist or change. The interventional therapy group still contains patients awaiting initiation or completion of treatment at the discretion of the local investigator team. Later outcome status might show whether those whose modified Rankin score is 2 or higher will undergo brain functional reorganisation cited in some brain 
arteriovenous malformations ${ }^{29}$ to the degrees known in focal brain infarcts. ${ }^{30}$ The range of potential outcome events and potential changes in the clinical status gathered over the coming 5 years should provide important clinical insights into the best long-term management of patients diagnosed with an unruptured brain arteriovenous malformation.

\section{Supplementary Material}

Refer to Web version on PubMed Central for supplementary material.

\section{Acknowledgments}

This paper is dedicated in memoriam to our colleague and friend William L Young, in recognition of his contribution to brain arteriovenous malformations research and to the ARUBA trial. We thank patients who participate in this trial. This trial is funded internationally by the National Institute of Neurological Diosorders and Stroke of the US National Institutes of Health via cooperative agreements U01NS051483 (CCC; J.P.Mohr, PI) and U01 NS051566 (DCC; A.J. Moskowitz, PI). No commercial funding sources have been involved. ARUBA has been officially adopted by the UK Stroke Research Network and endorsed by the Société Française Neurovasculaire (SFNV). Initial results have been presented at the European Stroke Conference; London, UK; May 28-31, 2013.

\section{References}

1. Al-Shahi R, Bhattacharya JJ, Currie DG, et al. the Scottish Intracranial Vascular Malformation Study Collaborators. Prospective, population-based detection of intracranial vascular malformations in adults: the Scottish Intracranial Vascular Malformation Study (SIVMS). Stroke. 2003; 34:116369. [PubMed: 12702837]

2. Stapf C, Mast H, Sciacca RR, et al. the New York Islands AVM Study Collaborators. The New York Islands AVM Study: design, study progress, and initial results. Stroke. 2003; 34:e29-33. [PubMed: 12690217]

3. Ondra SL, Troupp H, George ED, Schwab K. The natural history of symptomatic arteriovenous malformations of the brain: a 24-year follow-up assessment. J Neurosurg. 1990; 73:387-91. [PubMed: 2384776]

4. Halim AX, Johnston SC, Singh V, et al. Longitudinal risk of intracranial hemorrhage in patients with arteriovenous malformation of the brain within a defined population. Stroke. 2004; 35:1697702. [PubMed: 15166396]

5. Stapf C, Mast H, Sciacca RR, et al. Predictors of hemorrhage in patients with untreated brain arteriovenous malformation. Neurology. 2006; 66:1350-55. [PubMed: 16682666]

6. Choi JH, Mast H, Sciacca RR, et al. Clinical outcome after first and recurrent hemorrhage in patients with untreated brain arteriovenous malformation. Stroke. 2006; 37:1243-47. [PubMed: 16614321]

7. van Beijnum J, Lovelock CE, Cordonnier C, et al. the SIVMS Steering Committee and the Oxford Vascular Study. Outcome after spontaneous and arteriovenous malformation-related intracerebral haemorrhage: population-based studies. Brain. 2009; 132:537-43. [PubMed: 19042932]

8. van Beijnum J, van der Worp HB, Buis DR, et al. Treatment of brain arteriovenous malformations: a systematic review and meta-analysis. JAMA. 2011; 306:2011-19. [PubMed: 22068993]

9. Hartmann A, Mast H, Mohr JP, et al. Determinants of staged endovascular and surgical treatment outcome of brain arteriovenous malformations. Stroke. 2005; 36:2431-35. [PubMed: 16224095]

10. Stapf C, Mohr JP, Choi JH, Hartmann A, Mast H. Invasive treatment of unruptured brain arteriovenous malformations is experimental therapy. Curr Opin Neurol. 2006; 19:63-68. [PubMed: 16415679]

11. Davis SM, Donnan GA. Unruptured brain arteriovenous malformations: another asymptomatic conundrum. Stroke. 2007; 38:3312. [PubMed: 17962583]

12. Joint Writing Group of the Technology Assessment Committee American Society of Interventional and Therapeutic Neuroradiology, Joint Section on Cerebrovascular Neurosurgery a Section of the 
American Association of Neurological Surgeons and Congress of Neurological Surgeons, Section of Stroke and the Section of Interventional Neurology of the American Academy of Neurology et al. Reporting terminology for brain arteriovenous malformation clinical and radiographic features for use in clinical trials. Stroke. 2001; 32:1430-42. [PubMed: 11387510]

13. Dorman PJ, Slattery J, Farrell B, Dennis MS, Sandercock PAG. the United Kingdom Collaborators in the International Stroke Trial. A randomized comparison of the Euroquol and Short Form-36 after stroke. BMJ. 1997; 315:461. [PubMed: 9284664]

14. van Swieten JC, Koudstaal PJ, Visser MC, et al. Interobserver agreement for the assessment of handicap in stroke patients. Stroke. 1988; 19:604-07. [PubMed: 3363593]

15. Spetzler RF, Martin NA. A proposed grading system for arteriovenous malformations. J Neurosurg. 1986; 65:476-83. [PubMed: 3760956]

16. Ogilvy CS, Stieg PE, Awad I, et al. the Special Writing Group of the Stroke Council, and the American Stroke Association. . AHA Scientific Statement: recommendations for the management of intracranial arteriovenous malformations: a statement for healthcare professionals from a special writing group of the Stroke Council, American Stroke Association. Stroke. 2001; 32:145871. [PubMed: 11387517]

17. Al-Shahi R, Stapf C. The prognosis and treatment of arteriovenous malformations of the brain. Pract Neurol. 2005; 5:194-205.

18. Ross J, Al-Shahi Salman R. Interventions for treating brain arteriovenous malformations in adults. Cochrane Database Syst Rev. 2010; 7:CD003436. [PubMed: 20614434]

19. Wedderburn CJ, van Beijnum J, Bhattacharya JJ, et al. on behalf of the SIVMS Collaborators. Outcome after interventional or conservative management of unruptured brain arteriovenous malformations: a prospective, population-based cohort study. Lancet Neurol. 2008; 7:223-30. [PubMed: 18243054]

20. Laakso A, Dashti R, Seppänen J, et al. Long-term excess mortality in 623 patients with brain arteriovenous malformations. Neurosurgery. 2008; 63:244-53. [PubMed: 18797354]

21. Barnett HJ, Peerless SJ, McCormick CW. In answer to the question: “As compared to what?" A progress report on the EC/IC bypass study. Stroke. 1980; 11:137-40. [PubMed: 6989033]

22. Barnett HJ, Sackett D, Taylor DW, et al. Are the results of the extracranial-intracranial bypass trial generalizable? N Engl J Med. 1987; 26:316, 820-24.

23. Mohr JP, Moskowitz AJ, Stapf C, et al. The ARUBA trial: current status, future hopes. Stroke. 2010; 41:e537-40. [PubMed: 20634478]

24. Rothwell PM. External validity of randomised controlled trials: "To whom do the results of this trial apply? Lancet. 2005; 365:82-93. [PubMed: 15639683]

25. Executive Committee for the Asymptomatic Carotid Atherosclerosis Study. Endarterectomy for asymptomatic carotid artery stenosis. JAMA. 1995; 273:1421-28. [PubMed: 7723155]

26. Powers WJ, Clarke WR, Grubb RL, et al. for the COSS Investigators. Extracranial-intracranial bypass surgery for stroke prevention in hemodynamic cerebral ischemia. The Carotid Occlusion Surgery Study randomized trial. JAMA. 2011; 306:1983-92. [PubMed: 22068990]

27. Molyneux A, Kerr R, Stratton I, et al. for the International Subarachnoid Aneurysm Trial (ISAT) Collaborative Group. International Subarachnoid Aneurysm Trial (ISAT) of neurosurgical clipping versus endovascular coiling in 2143 patients with ruptured intracranial aneurysms: a randomised trial. Lancet. 2002; 360:1267-74. [PubMed: 12414200]

28. Mendelow AD, Gregson BA, Rowan EN, et al. for the STICH II Investigators. Early surgery versus initial conservative treatment in patients with spontaneous supratentorial lobar intracerebral haematomas (STICH II): a randomised trial. Lancet. 2013; 382:397-408. [PubMed: 23726393]

29. La Piana R, Bourassa-Blanchette S, Klein D, et al. Brain reorganization after endovascular treatment in a patient with a large arteriovenous malformation: the role of diagnostic and functional neuroimaging techniques. Interv Neuroradiol. 2013; 19:329-38. [PubMed: 24070082]

30. Mohr JP. Historical observations on functional reorganization. Cerebrovasc Dis. 2004; 18:258-59. [PubMed: 15331878] 


\section{Panel: Research in context}

\section{Systematic review}

In 2010, a systematic Cochrane review ${ }^{18}$ showed that there is no evidence from randomised trials with clear clinical outcomes comparing different interventional treatments for brain arteriovenous malformations compared with medical therapy alone. At the time, ARUBA has been identified as the only ongoing randomised clinical trial assessing the potential benefit of preventive lesion eradication in patients harbouring an unruptured brain arteriovenous malformation. We updated this review in September, 2013, searching the Cochrane Central Register of Controlled Trials, Medline (1966 to Sept 30, 2013), Embase (1980 to Sept 30, 2013), and PubMed (1951 to Sept 30, 2013) for studies published in English and continental European languages using the same search strategy as previously reported. ${ }^{18}$ We also searched international registers of clinical trials, the contents pages of relevant journals, and bibliographies of relevant articles for randomised trials of any or all of the interventions for brain arteriovenous malformations, compared with usual medical therapy, with relevant clinical outcome measures. Currently, ARUBA is the only randomised controlled clinical trial addressing this compelling question.

\section{Interpretation}

After 33 months of follow-up, the ARUBA data provide evidence that medical management alone is superior to preventive interventional management for the prevention of death or stroke in patients diagnosed with an unruptured brain arteriovenous malformation. Over the same follow-up period, the secondary endpoint analysis shows the risk of death and neurological disability is significantly lower for patients managed without intervention. Baseline characteristics of trial participants are much the same as those of representative population-based cohorts. The risk associated with preventive interventional therapy has proven to be higher than previously anticipated. The trial is continuing its observational phase for at least 5 more years to establish whether the disparities in clinical outcome will persist over time. 


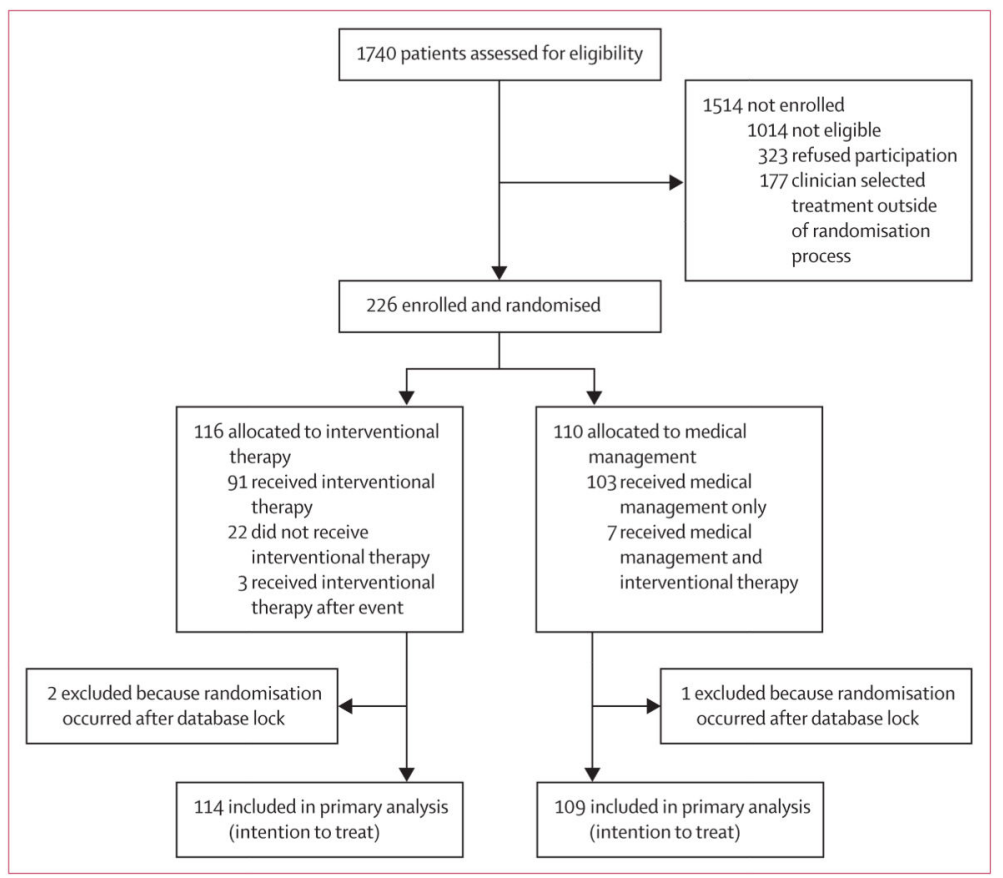

Figure 1.

Trial profile 


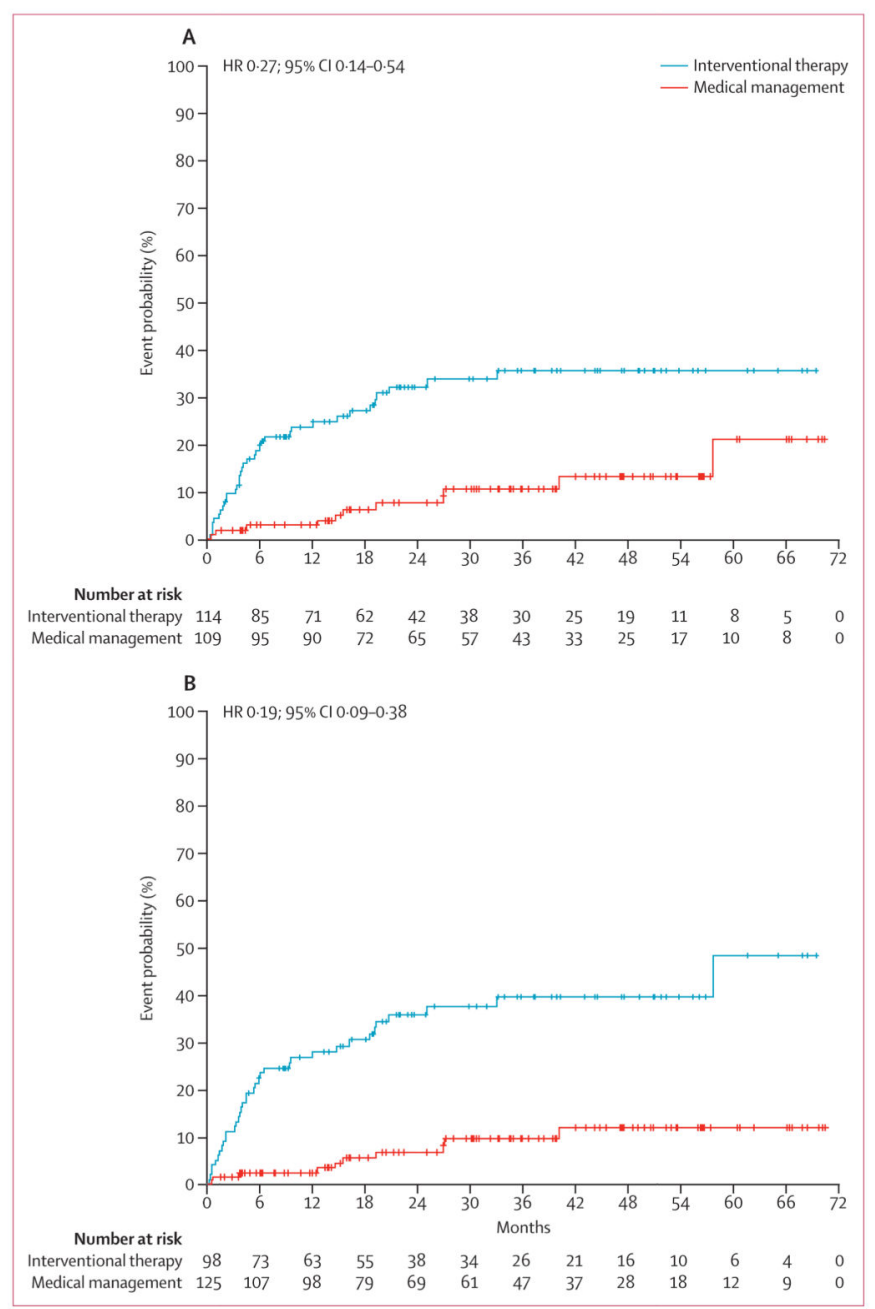

Figure 2. Kaplan-Meier estimated event rate by (A) as-randomised and (B) as-treated Crosses depict censored patients. HR=hazard ratio. (A) Log-rank $\chi^{2}=16 \cdot 07 ; \mathrm{p}<0 \cdot 0001$. (B) Log-rank $\chi^{2}=26 \cdot 9, \mathrm{p}<0 \cdot 0001$. Seven patients in the medical management crossed over to interventional therapy. 20 patients in the interventional therapy group did not receive interventional therapy and are deemed crossovers to medical management. Three additional patients randomised to interventional therapy had a stroke before the initiation of interventional therapy and were placed in the medical management group for the as-treated analysis. 
Table 1

Baseline characteristics

\begin{tabular}{|c|c|c|}
\hline & Interventional therapy $(\mathrm{N}=114)$ & Medical management $(\mathrm{N}=109)$ \\
\hline Age (years) & $45(12)$ & $44(12)$ \\
\hline Women & $48(42 \%)$ & $44(40 \%)$ \\
\hline White & $98(86 \%)$ & $87(80 \%)$ \\
\hline Right-handed & $109(96 \%)$ & $100(92 \%)$ \\
\hline \multicolumn{3}{|l|}{ Clinical presentation } \\
\hline Seizure & $50(44 \%)$ & $45(41 \%)$ \\
\hline Headaches & $56(49 \%)$ & $59(54 \%)$ \\
\hline Focal deficit & $21(18 \%)$ & $10(9 \%) \mathrm{x}$ \\
\hline Other & $3(3 \%)$ & $8(7 \%)$ \\
\hline Asymptomatic & $44(39 \%)$ & $49(45 \%)$ \\
\hline \multicolumn{3}{|l|}{ Modified Rankin score } \\
\hline 0 & $55(48 \%)$ & $51(47 \%)$ \\
\hline 1 & $59(52 \%)$ & $58(53 \%)$ \\
\hline \multicolumn{3}{|l|}{ Spetzler-Martin grading score ${ }^{*}$} \\
\hline I & $32(29 \%)$ & $33(30 \%)$ \\
\hline II & $44(39 \%)$ & $27(25 \%)$ \\
\hline III & $28(25 \%)$ & $34(31 \%)$ \\
\hline IV & $8(7 \%)$ & $15(14 \%)$ \\
\hline \multicolumn{3}{|l|}{ AVM morphology } \\
\hline Maximum AVM size (mm) & $25(12)$ & $28(11)$ \\
\hline AVM size $<3 \mathrm{~cm}$ & $78(68 \%)$ & $60(55 \%)$ \\
\hline AVM side, left & $48(42 \%)$ & $50(46 \%)$ \\
\hline Lobar AVM location & $104(91 \%)$ & $99(91 \%)$ \\
\hline Infratentorial AVM location & $7(6 \%)$ & $5(5 \%)$ \\
\hline Eloquent AVM location ${ }^{\dagger}$ & $54(47 \%)$ & $51(47 \%)$ \\
\hline \multicolumn{3}{|l|}{ Concurrent arterial aneurysms } \\
\hline Associated aneurysm ${ }^{*}$ & $15(13 \%)$ & $21(19 \%)$ \\
\hline Unrelated aneurysm & $4(4 \%)$ & $7(6 \%)$ \\
\hline \multicolumn{3}{|l|}{ Venous drainage pattern ${ }^{*}$} \\
\hline Superficial only & $78(70 \%)$ & $69(63 \%)$ \\
\hline Any deep & $34(30 \%)$ & $40(37 \%)$ \\
\hline
\end{tabular}

Data are mean (SD) or number (\%). AVM=arteriovenous malformation.

* Unavailable for two patients in the interventional therapy group who were enrolled without angiography. 
${ }^{\dagger}$ Eloquent is any AVM location involving the sensorimotor, language, or visual cortex; the hypothalamus and thalamus; the internal capsule; the brainstem; the cerebellar peduncles; or the deep cerebellar nuclei. 15

Associated arterial aneurysms are flow-related aneurysms located on a feeding artery or within the AVM nidus (so-called intranidal aneurysms). ${ }^{12}$ 
Table 2

Stroke and mortality by randomisation assignment*

\begin{tabular}{|c|c|c|c|}
\hline & Interventional therapy $(n=114)$ & Medical management (n=109) & Risk ratio $(95 \%$ CI) \\
\hline Death or stroke & $35(30 \cdot 7 \%)$ & $11(10 \cdot 1 \%)$ & $0.33(0 \cdot 18-0 \cdot 61)$ \\
\hline \multicolumn{4}{|l|}{ Death } \\
\hline All cause & $3(2 \cdot 6 \%)$ & $2(1.8 \%)$ & $0 \cdot 70(0 \cdot 12-4 \cdot 09)$ \\
\hline AVM-related & $2(1 \cdot 8 \%)$ & 0 & .. \\
\hline \multicolumn{4}{|l|}{ First stroke } \\
\hline All & $34(29 \cdot 8 \%)$ & $9(8.3 \%)$ & $0 \cdot 28(0 \cdot 14-0 \cdot 55)$ \\
\hline Haemorrhagic & $25(21.9 \%)$ & $6(5 \cdot 5 \%)$ & $0 \cdot 25(0 \cdot 11-0 \cdot 59)$ \\
\hline Ischaemic & $9(7 \cdot 9 \%)$ & $3(2 \cdot 8 \%)$ & $0 \cdot 35(0 \cdot 10-1 \cdot 25)$ \\
\hline
\end{tabular}

Data are number of patients (\%). AVM=arteriovenous malformation.

* Primary outcome events only. 
Table 3

Stroke and mortality as treated*

\begin{tabular}{|c|c|c|c|}
\hline & Interventional therapy $(\mathrm{n}=98)$ & Medical management (n=125) & Risk ratio $(95 \% \mathrm{CI})$ \\
\hline Death or stroke & $36(36 \cdot 7 \%)$ & $10(8 \cdot 0 \%)$ & $0 \cdot 22(0 \cdot 11-0 \cdot 42)$ \\
\hline \multicolumn{4}{|l|}{ Death } \\
\hline All cause & $3(3 \cdot 1 \%)$ & $2(1.6 \%)$ & $0.52(0 \cdot 09-3 \cdot 07)$ \\
\hline AVM-related & $2(2 \cdot 0 \%)$ & 0 & .. \\
\hline \multicolumn{4}{|l|}{ First stroke } \\
\hline All & $35(35 \cdot 7 \%)$ & $8(6 \cdot 4 \%)$ & $0 \cdot 18(0 \cdot 09-0 \cdot 37)$ \\
\hline Haemorrhagic & $24(24 \cdot 5 \%)$ & $7(5 \cdot 6 \%)$ & $0 \cdot 23(0 \cdot 10-0 \cdot 51)$ \\
\hline Ischaemic & $11(11 \cdot 2 \%)$ & $1(0 \cdot 8 \%)$ & $0 \cdot 07(0 \cdot 10-0 \cdot 54)$ \\
\hline
\end{tabular}

Data are number of patients (\%). AVM=arteriovenous malformation.

* Primary outcome events only. 


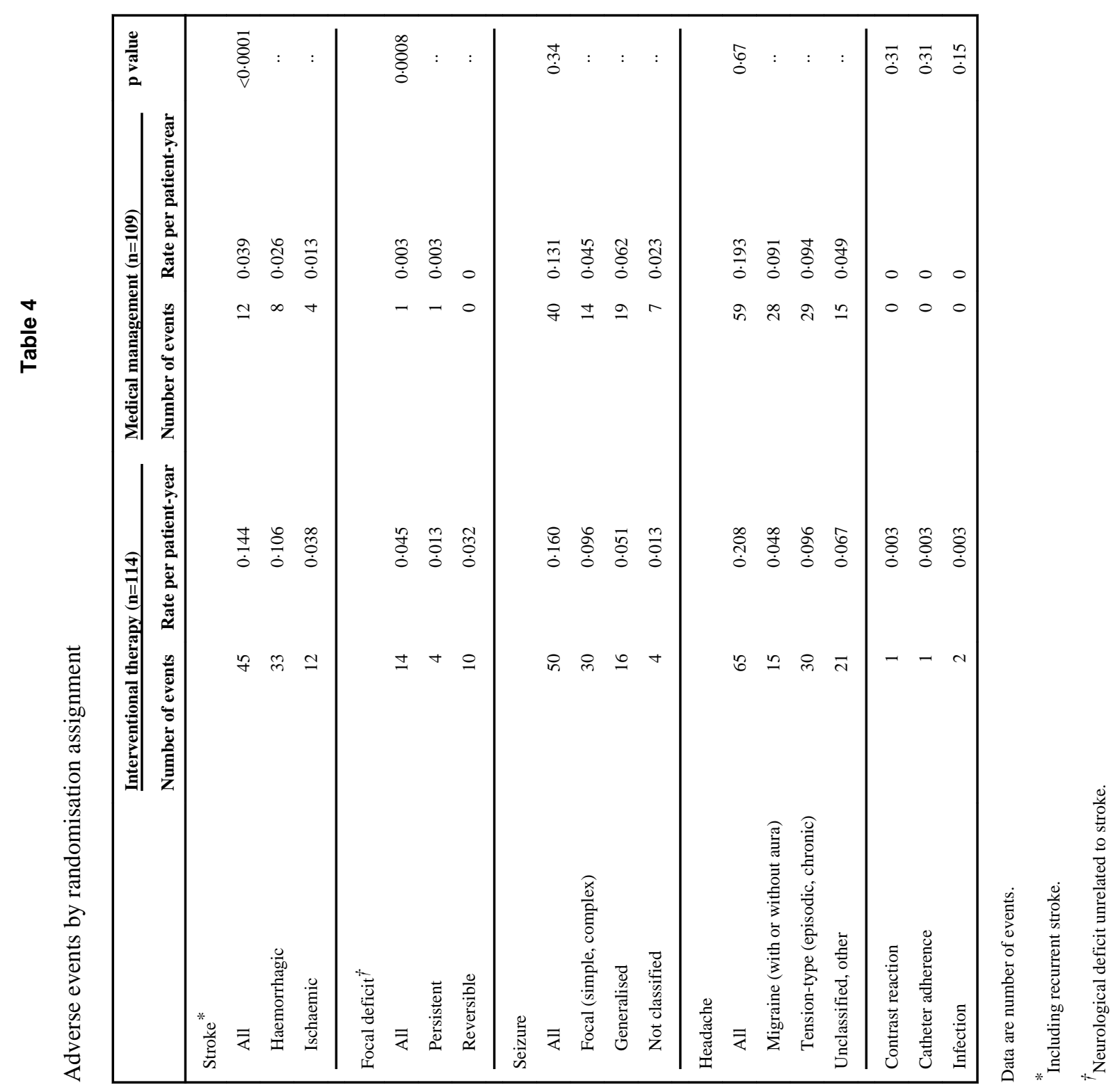

Lancet. Author manuscript; available in PMC 2015 February 15. 